\title{
SPACE STATION \\ SOLAR ARRAY JOINT REPAIR
}

Stuart Loewenthal ${ }^{\star}$, Curtis Allmon*, Carter Reznik**, Justin McFatter ${ }^{\star \star}$ and Robert E. Davis ${ }^{\star \star *}$

*Lockheed Martin Space Systems Company, Sunnyvale CA **Boeing Space Systems, Houston, TX

${ }^{* \star \star}$ NASA Johnson Space Center, Houston, TX

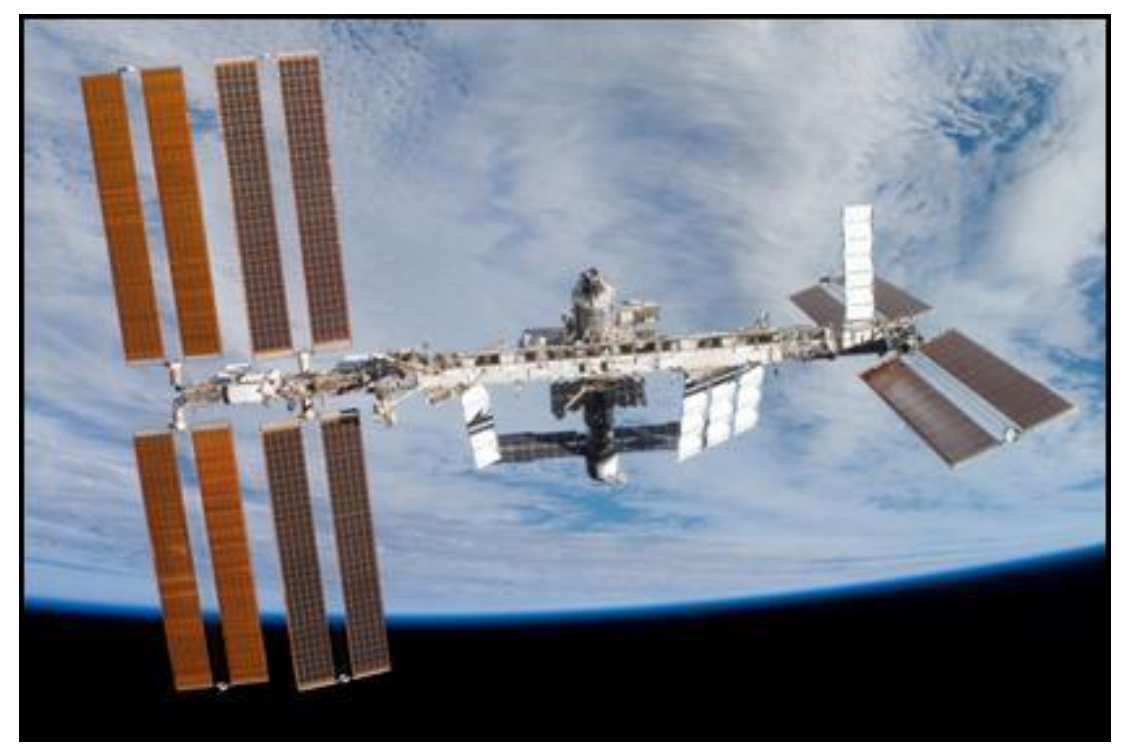




\section{Solar Alpha Rotary Joint (SARJ)}

L OCK HEE D MARTIN

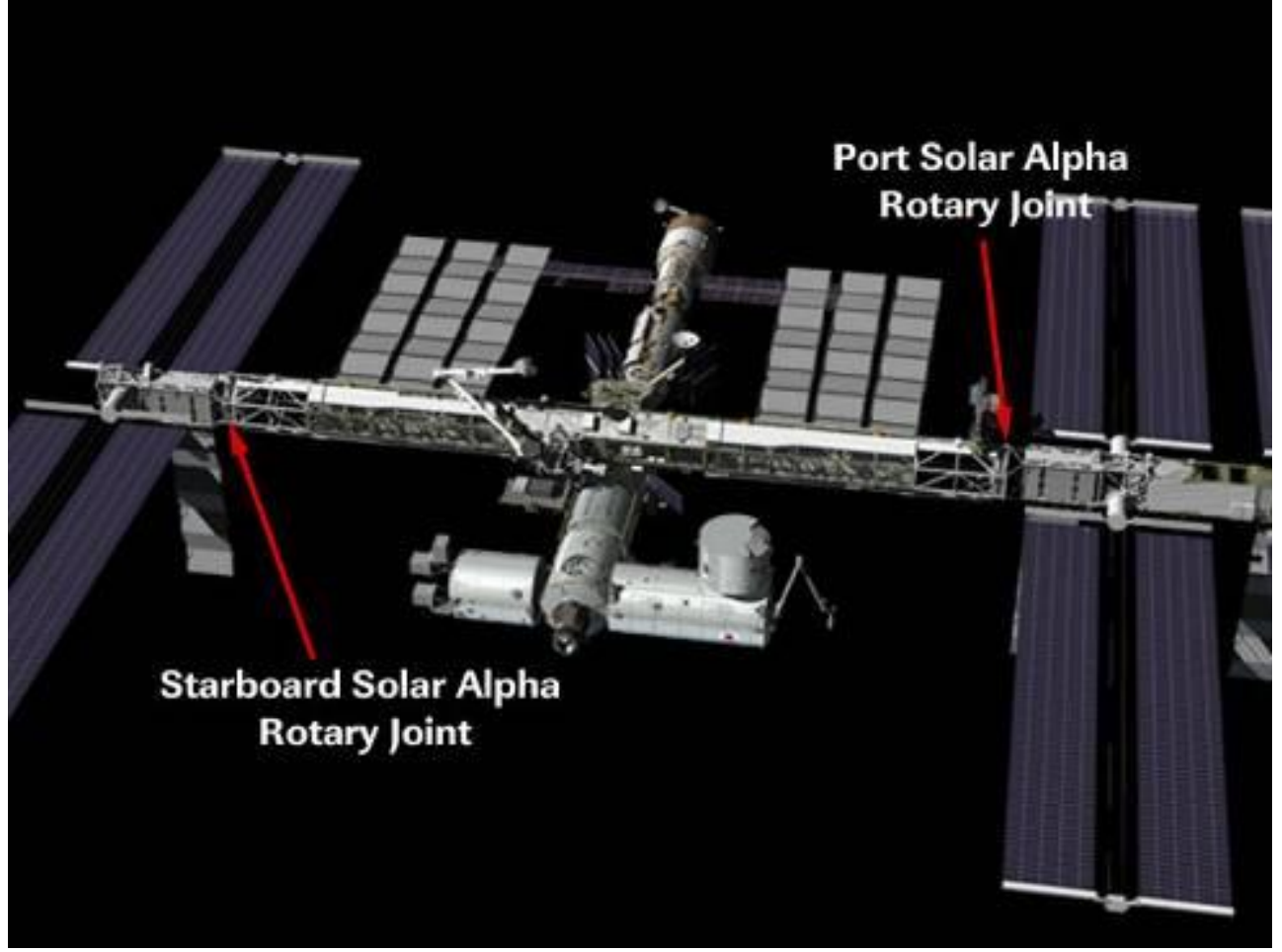

- SARJ orients port \& starboard solar array wings to sun providing power to station

- 12 Trundle Bearing Assemblies support a 10.5 ft diameter race rings (redundant)

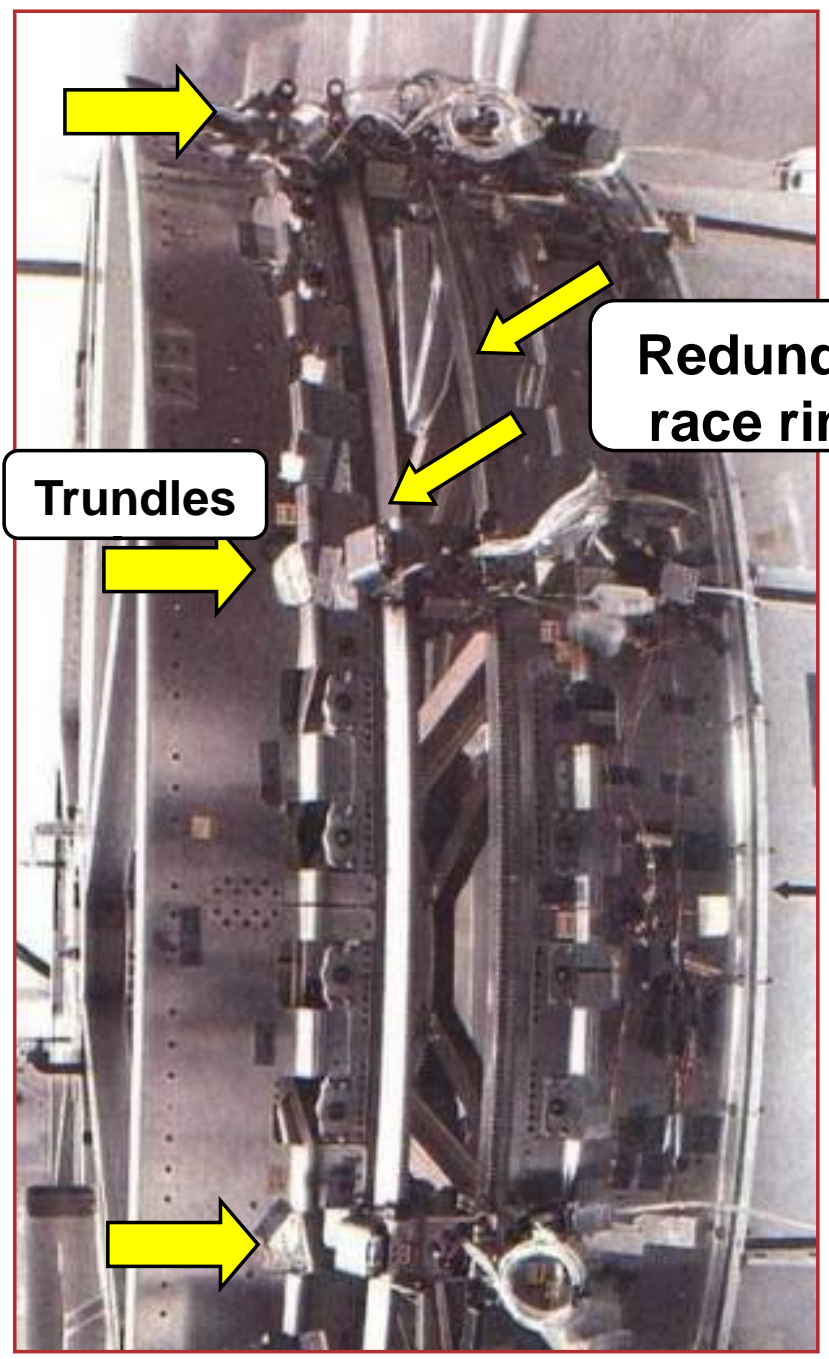




\section{SARJ Trundle Bearing Assys \& Race Ring $\mathbb{Q}_{\text {WDEINE }}$}

cocknto wartion

\section{Candidate Bearing Configurations}
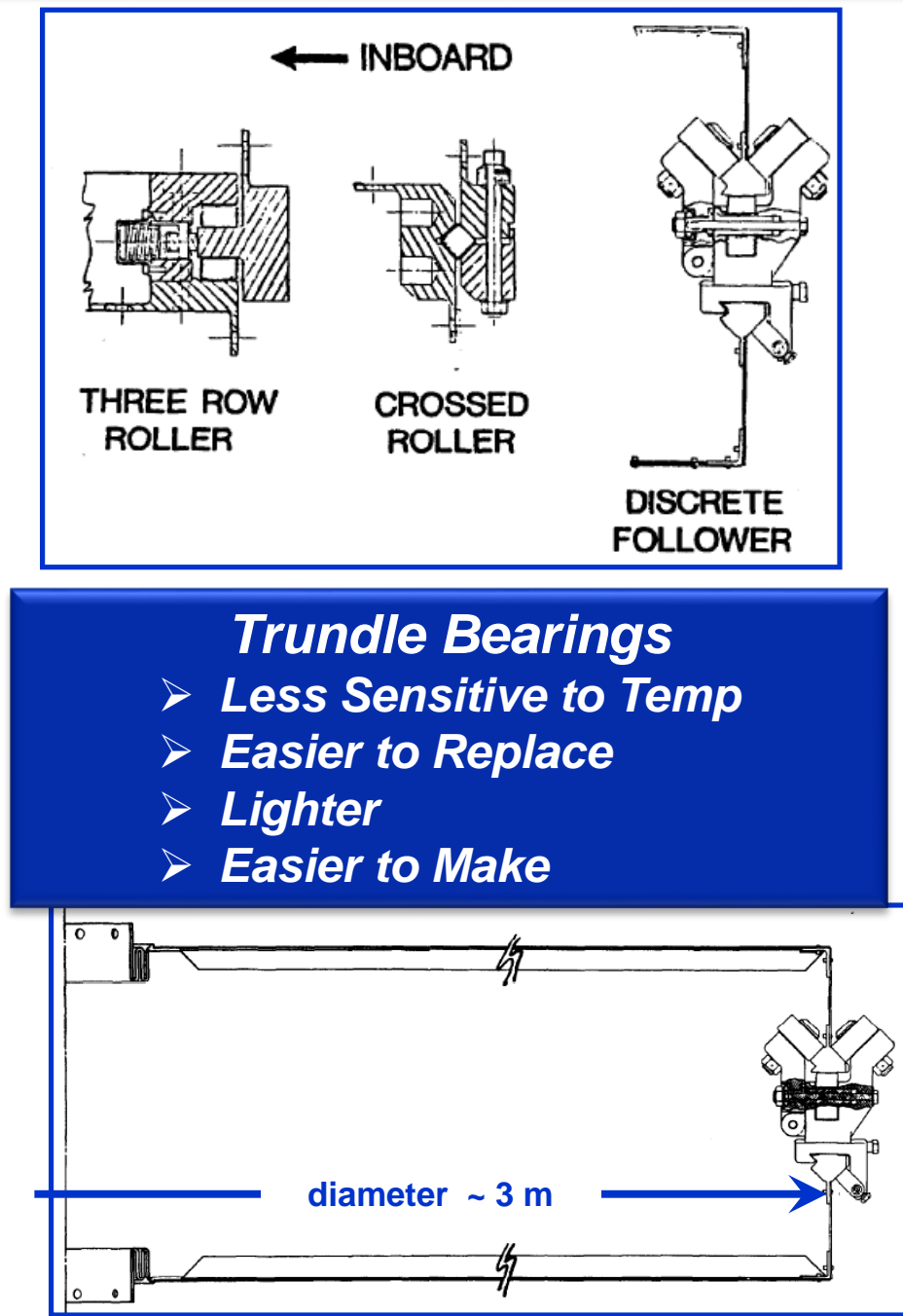

Camber

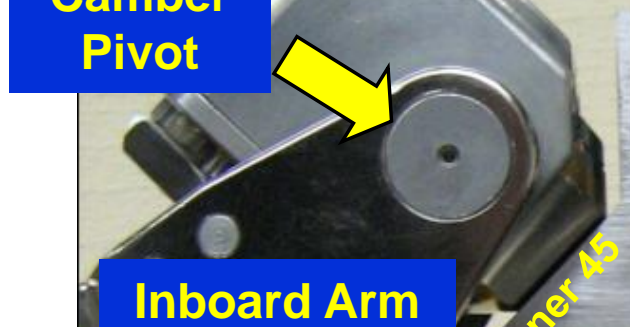

O

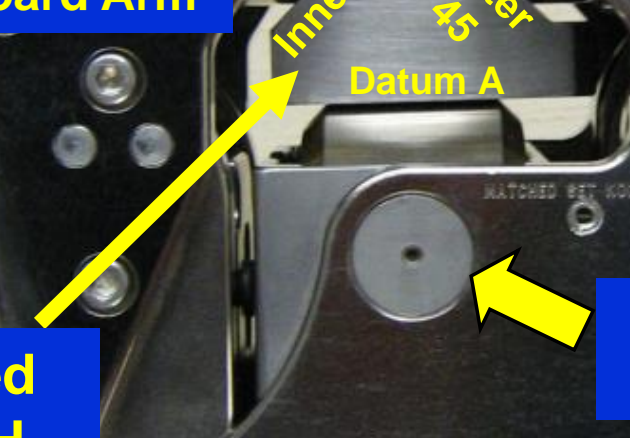

Nitrided 15-5PH

Race Ring

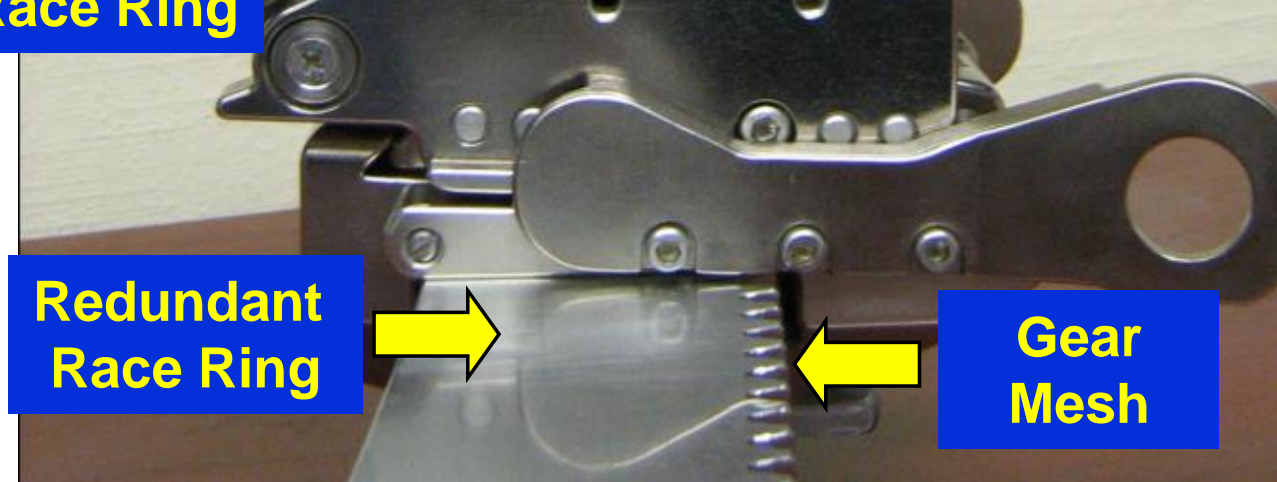




\section{The Problem}

- On 22 Oct 2007, ISS crew noticed vibration on camera in vicinity of Starboard SARJ

- Unit had less than 4 months running time

Starboard SARJ Day 245 - 250

RJMC Velocity Error Voltage Statistical Analysis by Joint Angle

- Velocity trace showed irregular servo errors at several clock positions

- Crew sent out to inspect and found race damage on outer 45 deg track

- Inner 45 deg \& Datum-A races Okay

- Port SARJ races Okay $\approx 14$ months at time of inspection

Starboard SARJ

velocity error changing each day

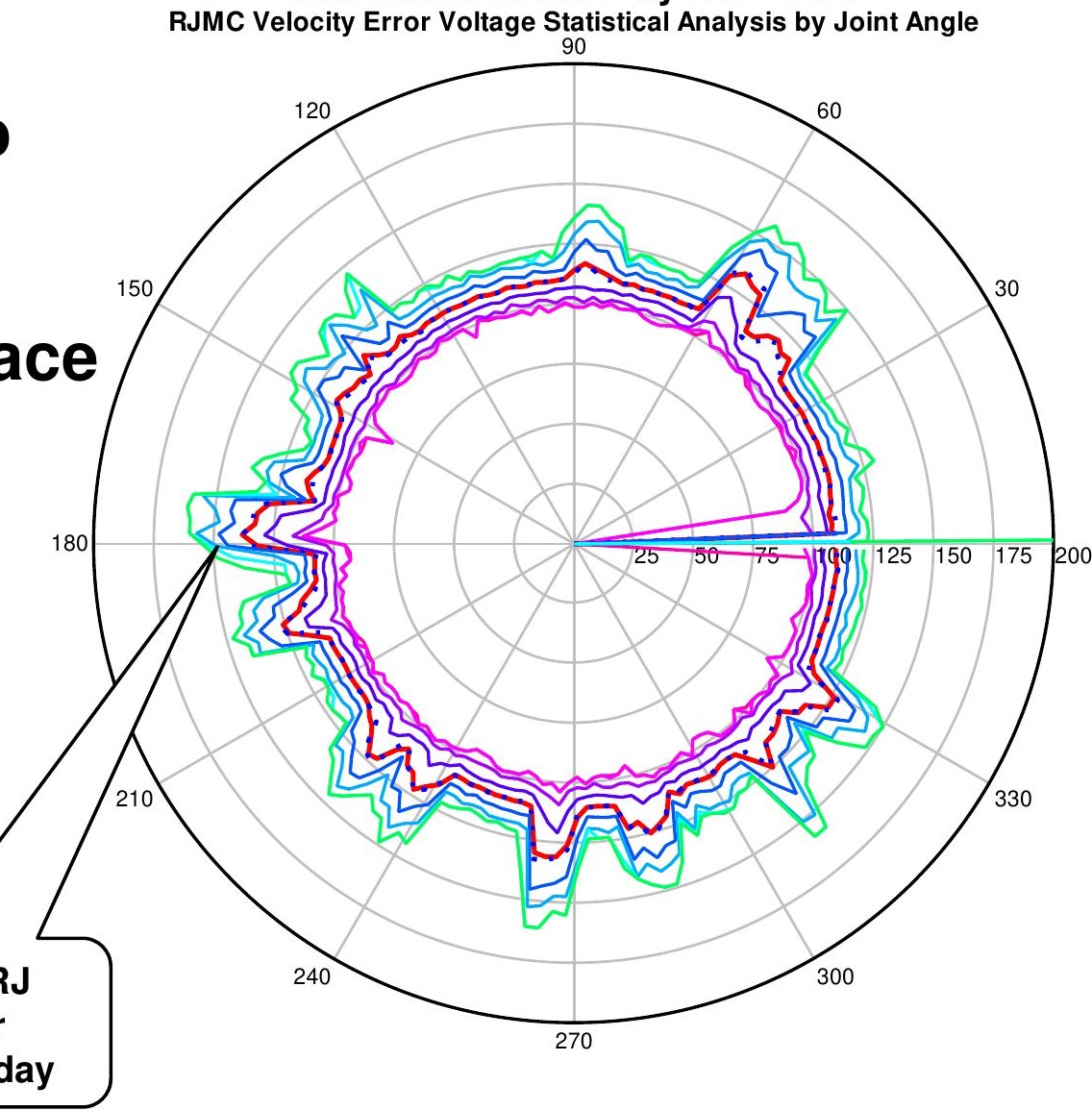




\section{Outer 45 Deg Race Shows Significant Damage}
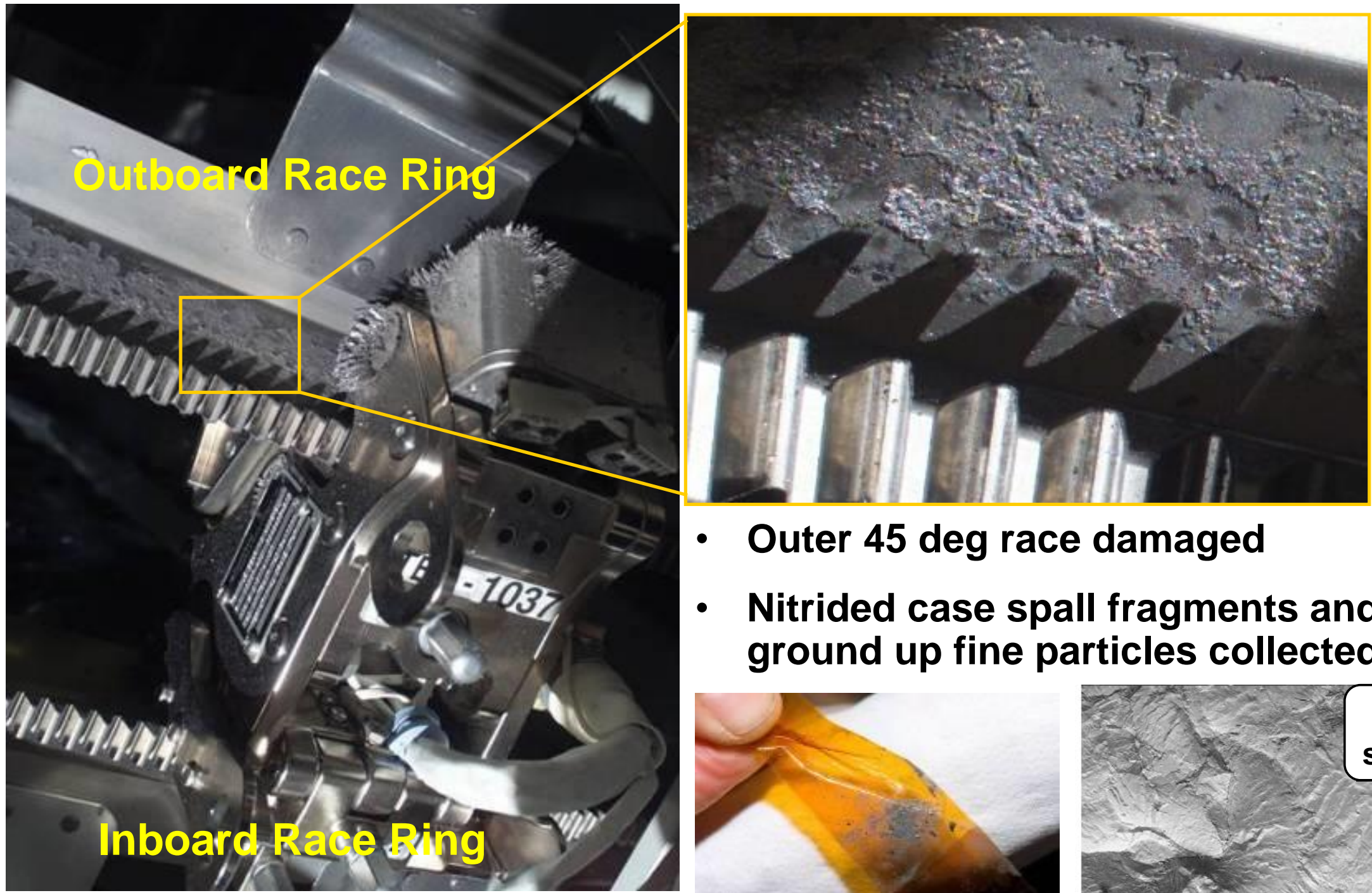

- Outer 45 deg race damaged

- Nitrided case spall fragments and ground up fine particles collected
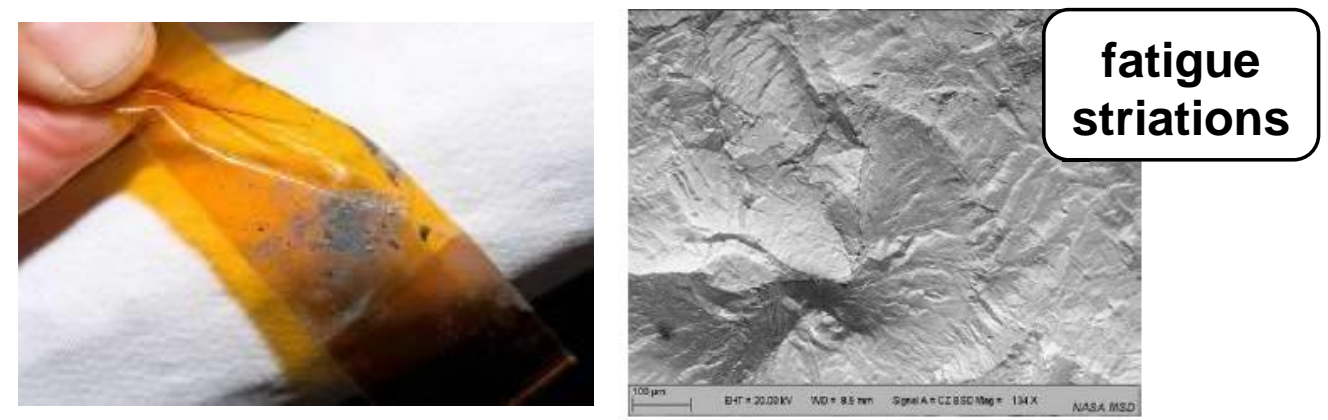


\section{Tipping Mechanism}

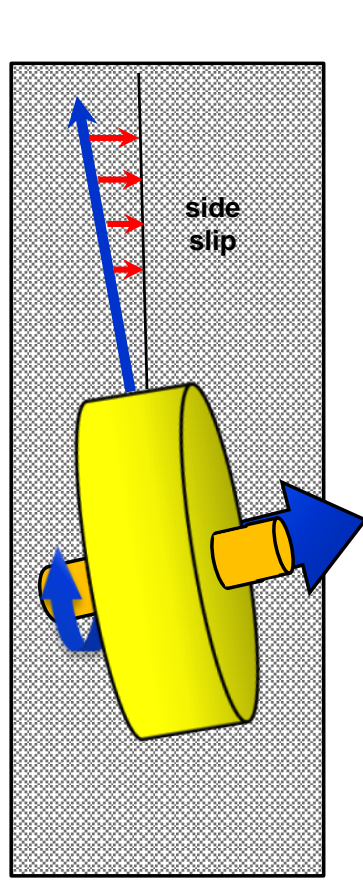

misaligned roller creates side slip between roller \& race

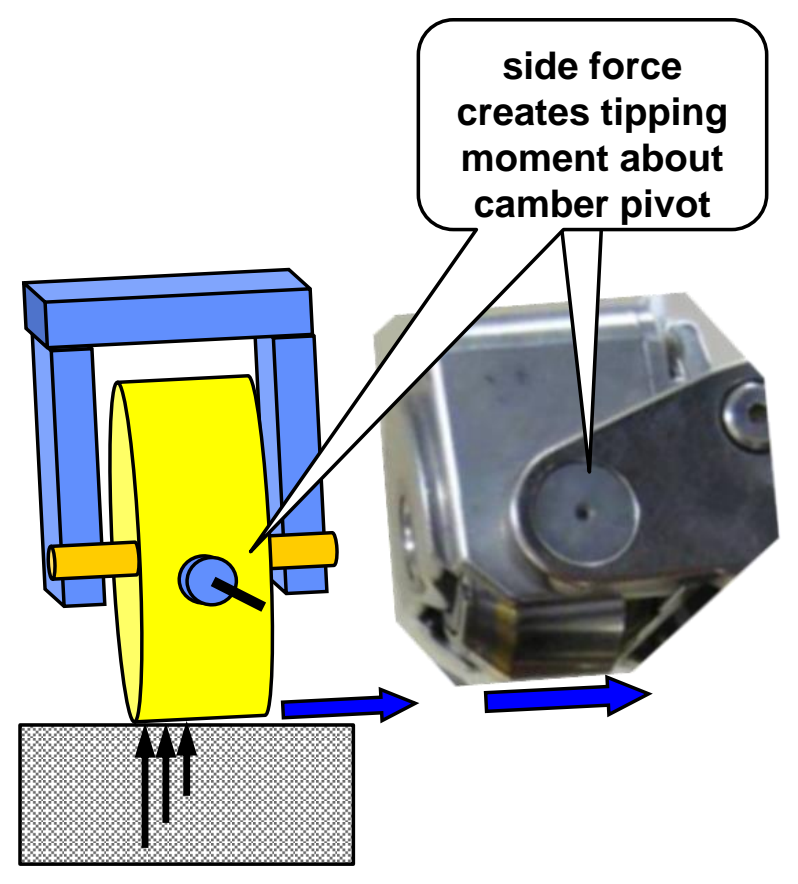

tipped roller creates high edge contact stress

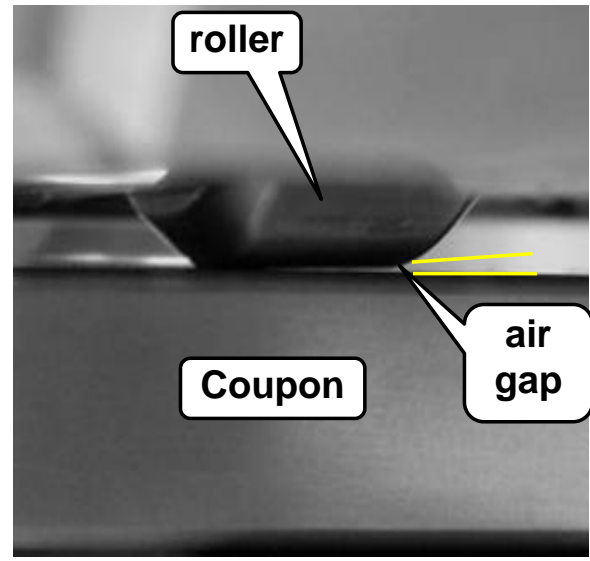

tipping confirmed

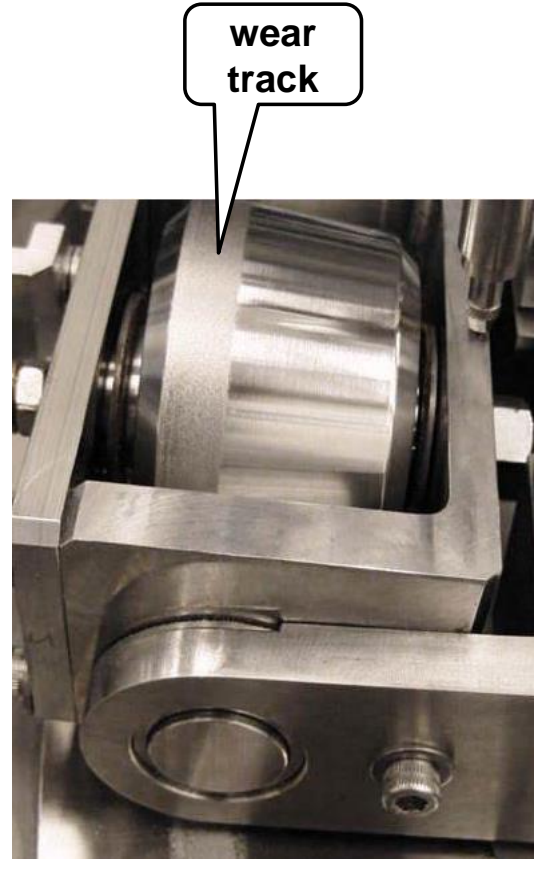

wear on edge of mistracked roller

Side force from misalignment plus high friction creates tipping and high contact edge stress 


\section{Failure Investigation}

L O C K H EE D MARTIN

fragments

- Government -Industry team formed to determine root cause

- Root cause found to be roller misalignment (aka mistracking) coupled with high contact friction

- generates tipping and high roller edge contact stresses

- edge stresses high enough to crack/fatigue the nitrided case

- intergranular networking (crack-like separations) likely contributed to damage

- Least difficult repair would be to lubricate the SARJ tracks with grease

- How do we validated this fix and how long will it last between regreasing?

- build a high fidelity SARJ life test rig
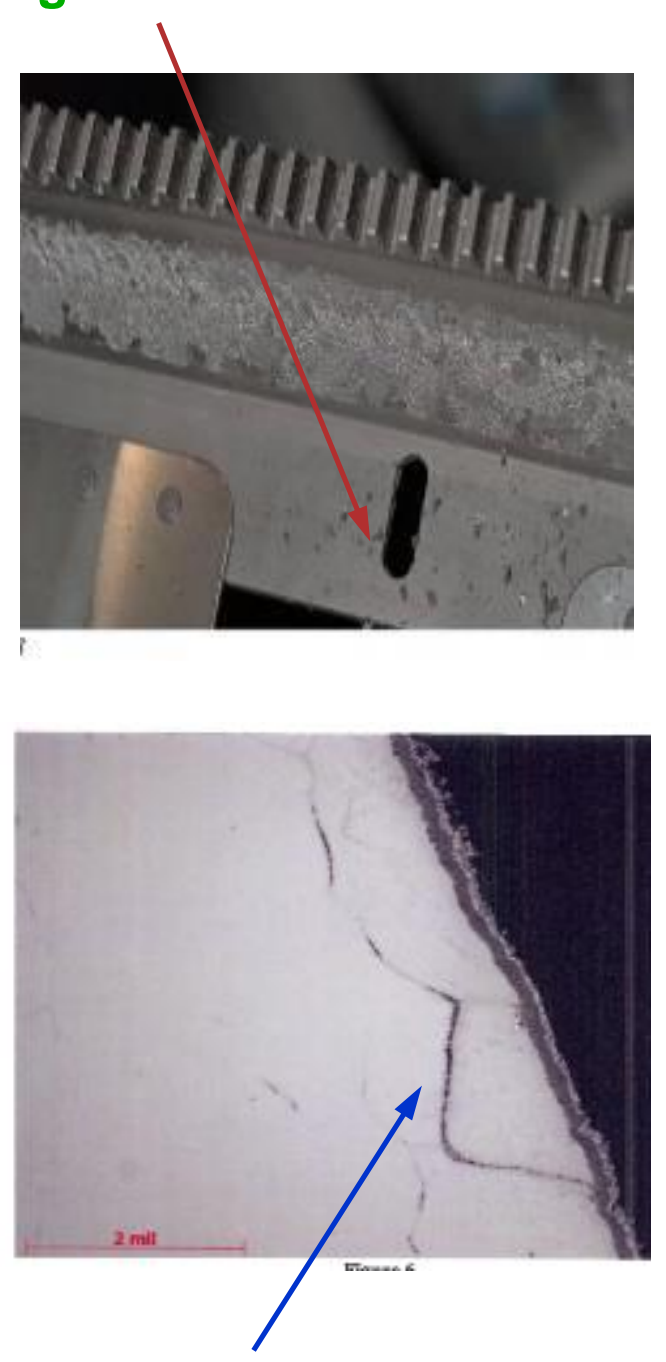


\section{Test Rig Parameters}

- Test rings diameters scaled by factor $\sim 3$ from flight

- able to fit into a reasonably sized vacuum chamber

- 3 full size, flight-like trundle roller assemblies selected vs normal 12.

- Track diameter sized to keep roller spacing same as flight

- Test plate is flight-like (same materials, heat-treats, surface finish)

- Outer track simulates damaged 45 deg outboard starboard flight race

- Inner track simulates undamaged Datum A roller contact

- Rollers have flight diameters, hardness, surface finish and profiles

- Mistracking angle set to $0.37 \mathrm{deg}$ high-nominal

- Test Rig runs $24 / 7$ with continuous data recording \& safety shutdowns

- Side friction, drag \& tipping angle recorded

- as indicator of lubrication degradation

- provides on-orbit health trending data

- trigger limits for rig safety shutdown

- Vacuum (<10-5 torr) at ambient temp

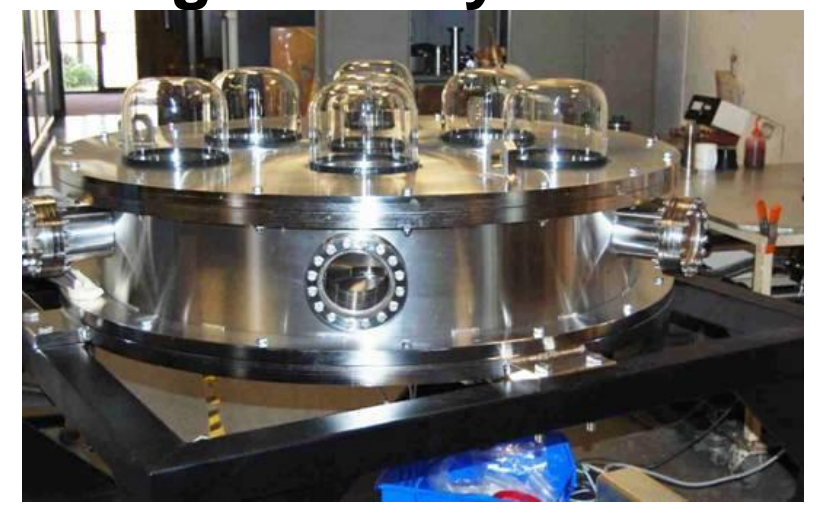




\section{Proving the Race will Last with Grease}

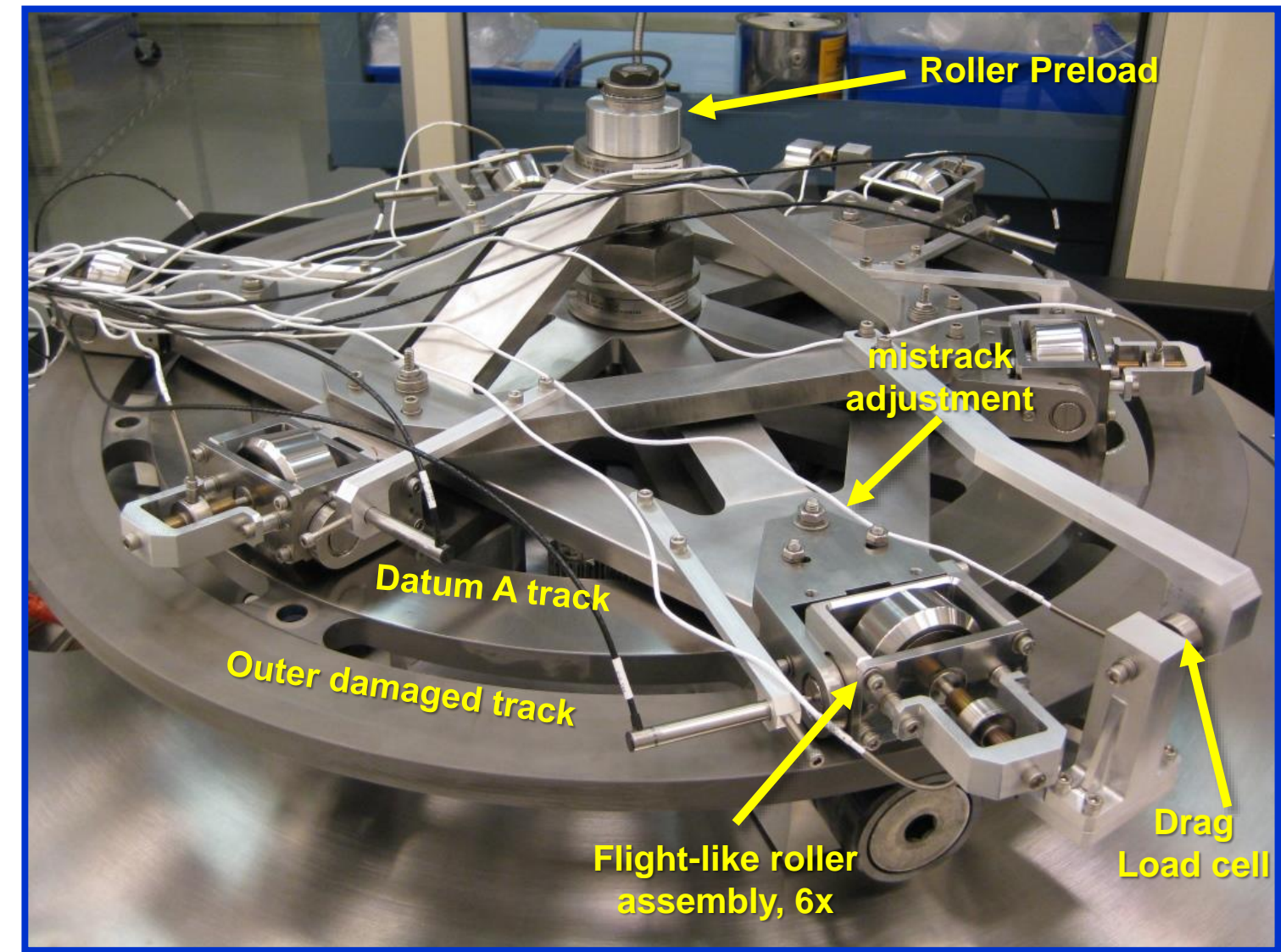

SARJ LITE Test Rig outside of vacuum chamber

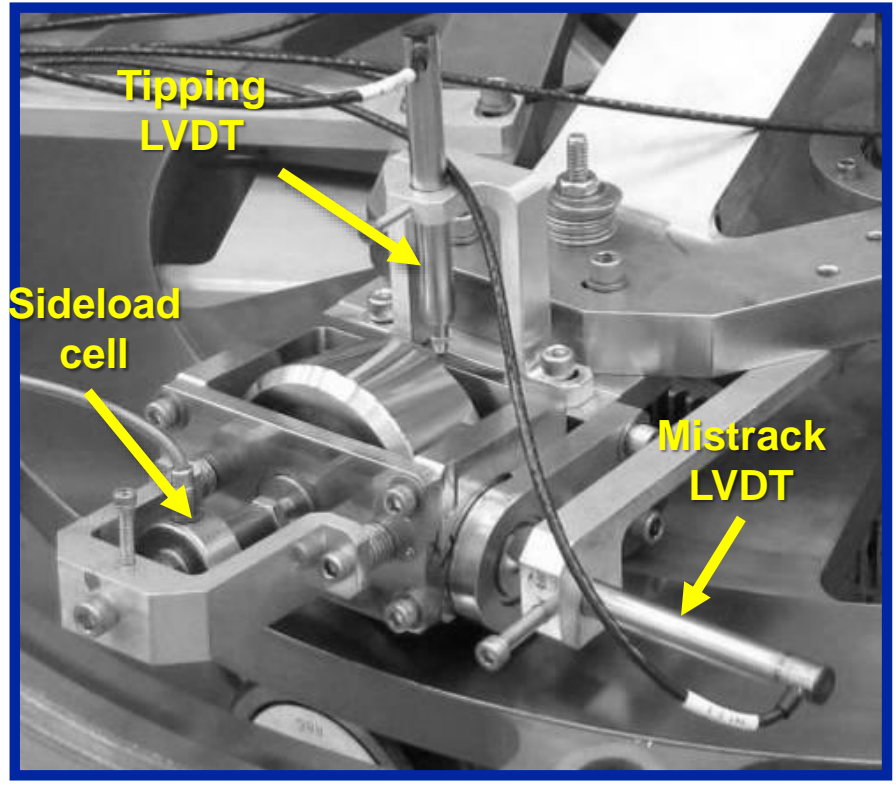

Each Roller Trundle Assembly is Well Instrumented 


\section{Outer and Inner Tracks Simulate On-Orbit SARJ NAsA}
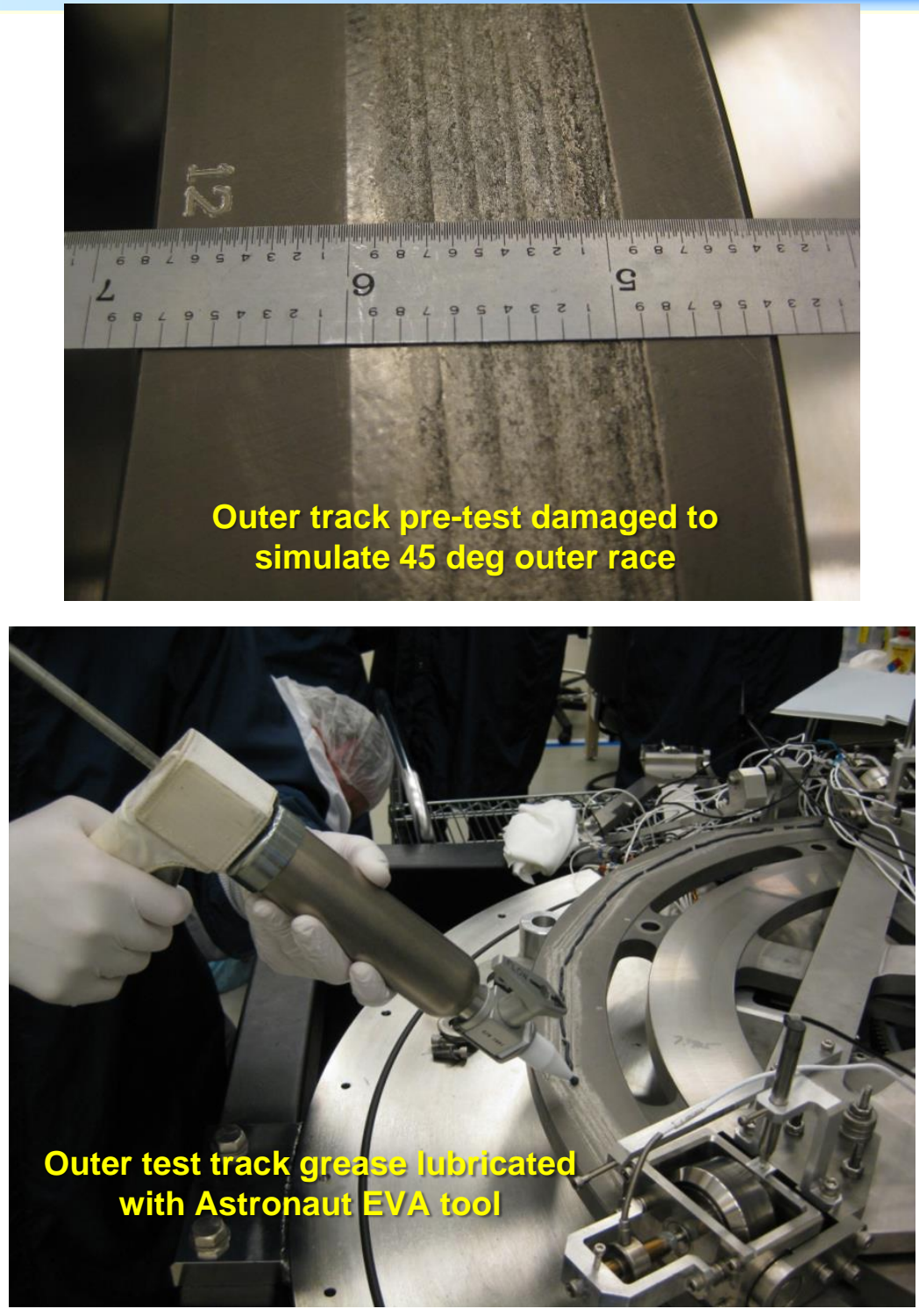
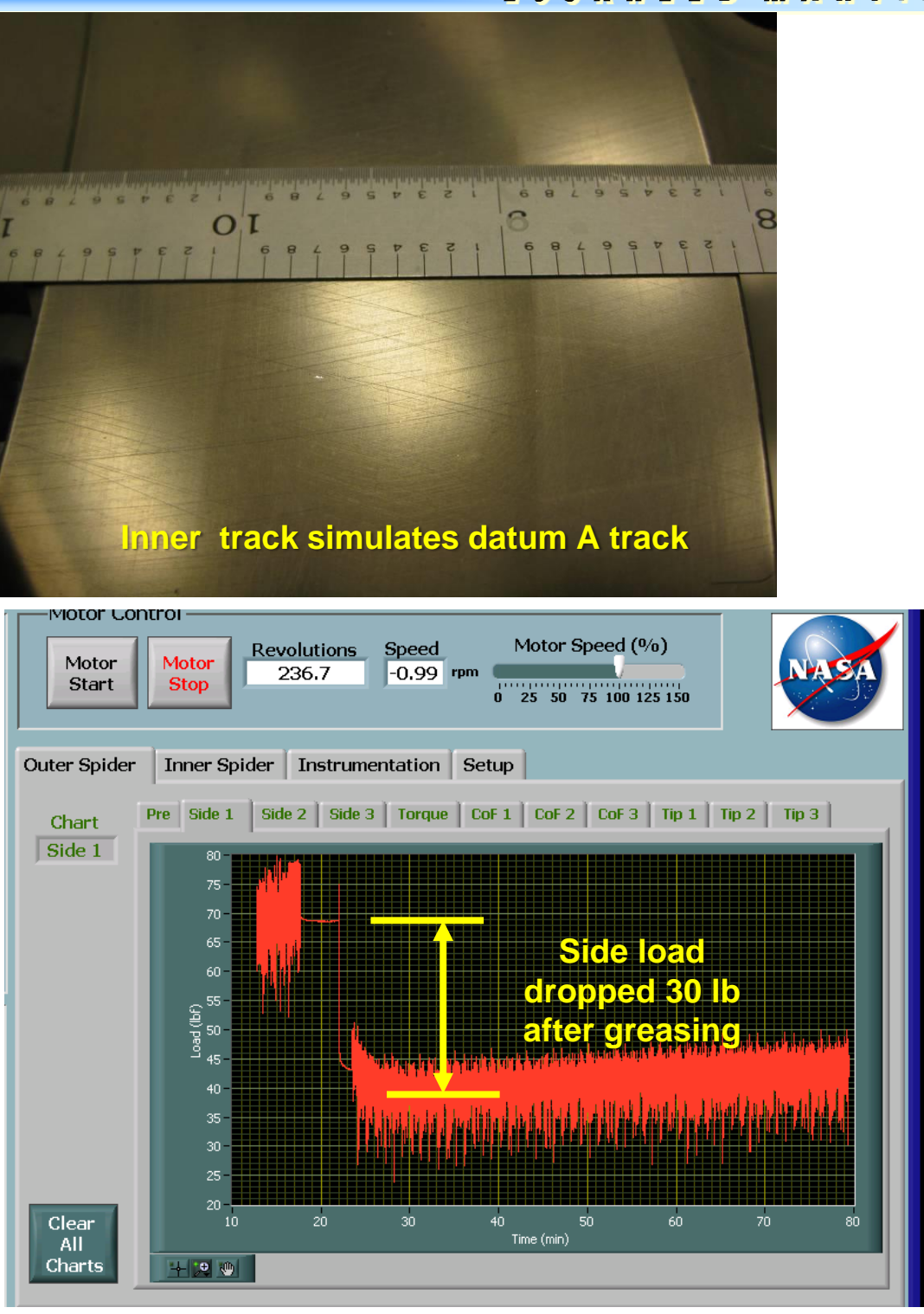


\section{Test Tracks at End of Test}

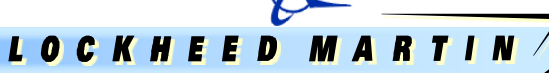

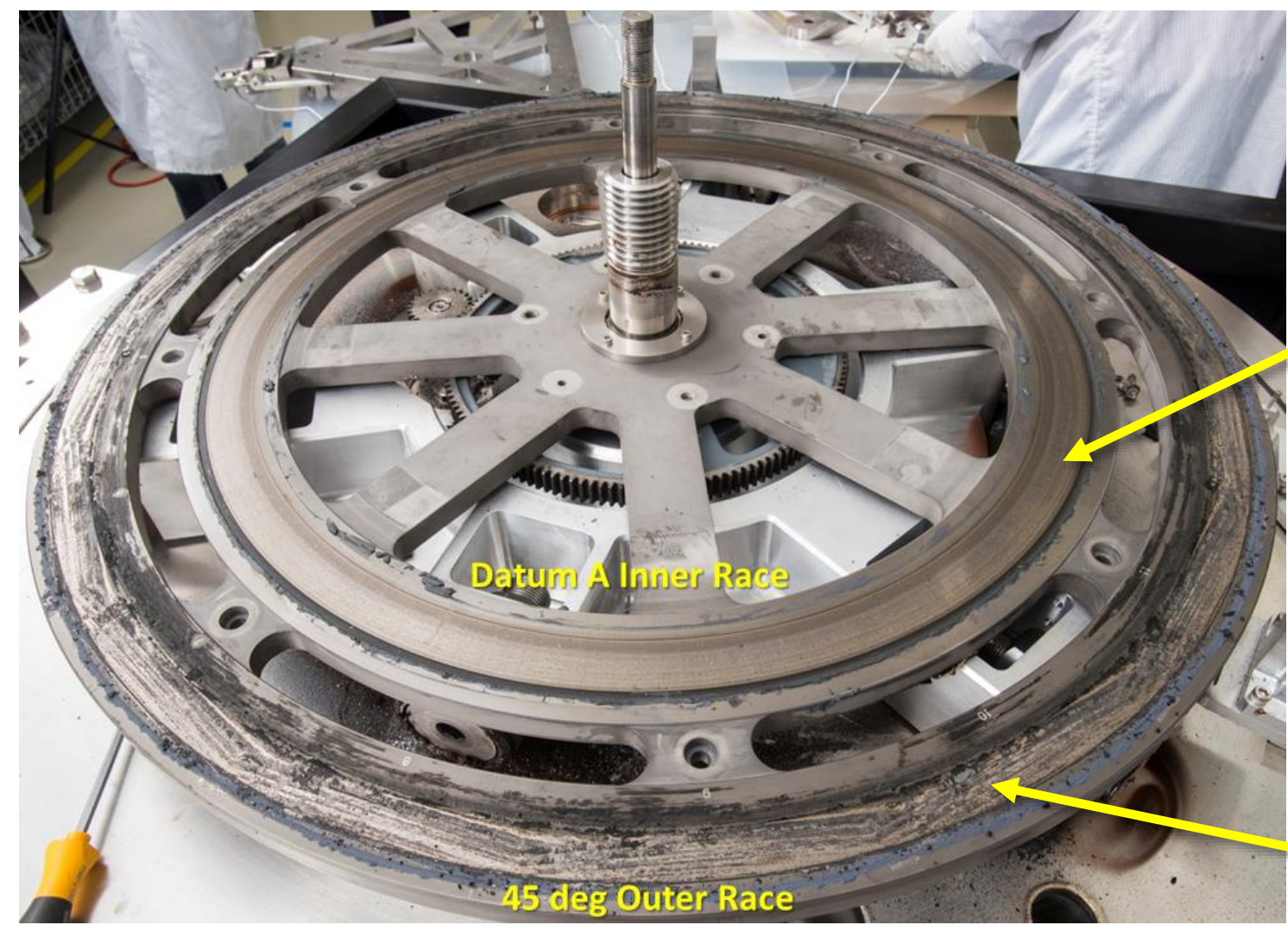

Inner and outer test tracks

Both Tracks showed Minor Wear after 36+ Equivalent On-orbit Years

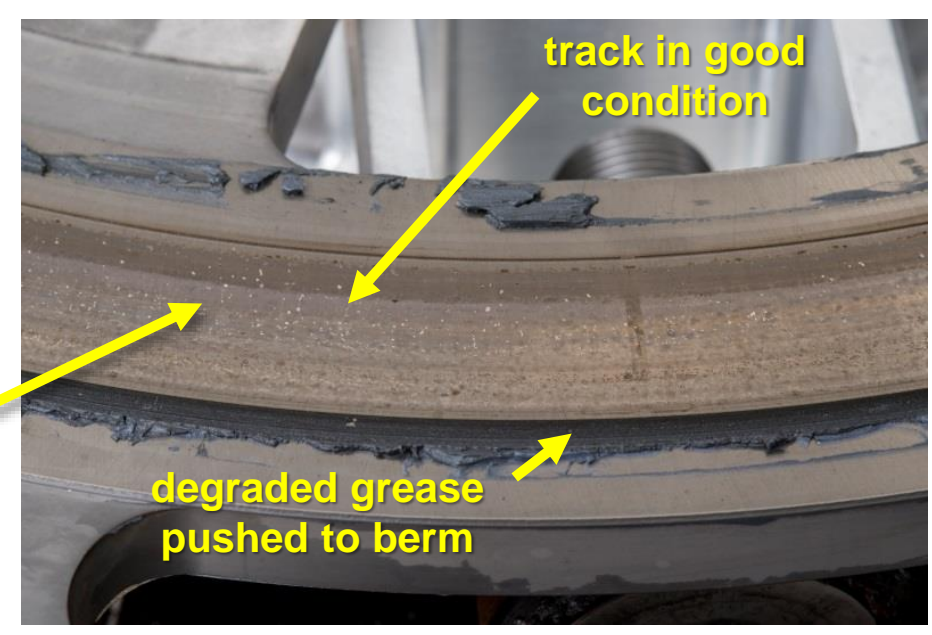

Datum A inner track

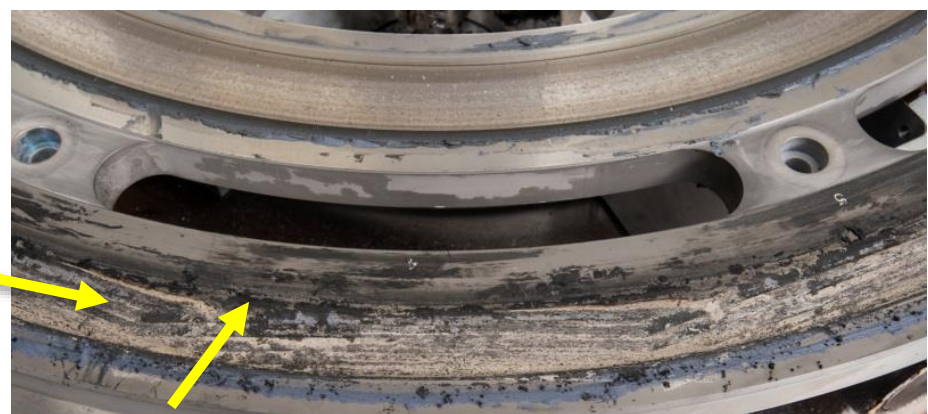

Degraded grease in pre-test damaged furciows.

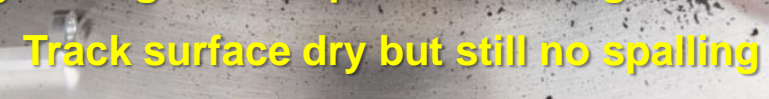
45 deg outer track 


\section{Post Script: \\ SARJ Races Greased (STS-126 Nov 2008)}

- Rollers replaced on damaged SARJ

- Starboard \& Port Races cleaned \& greased.

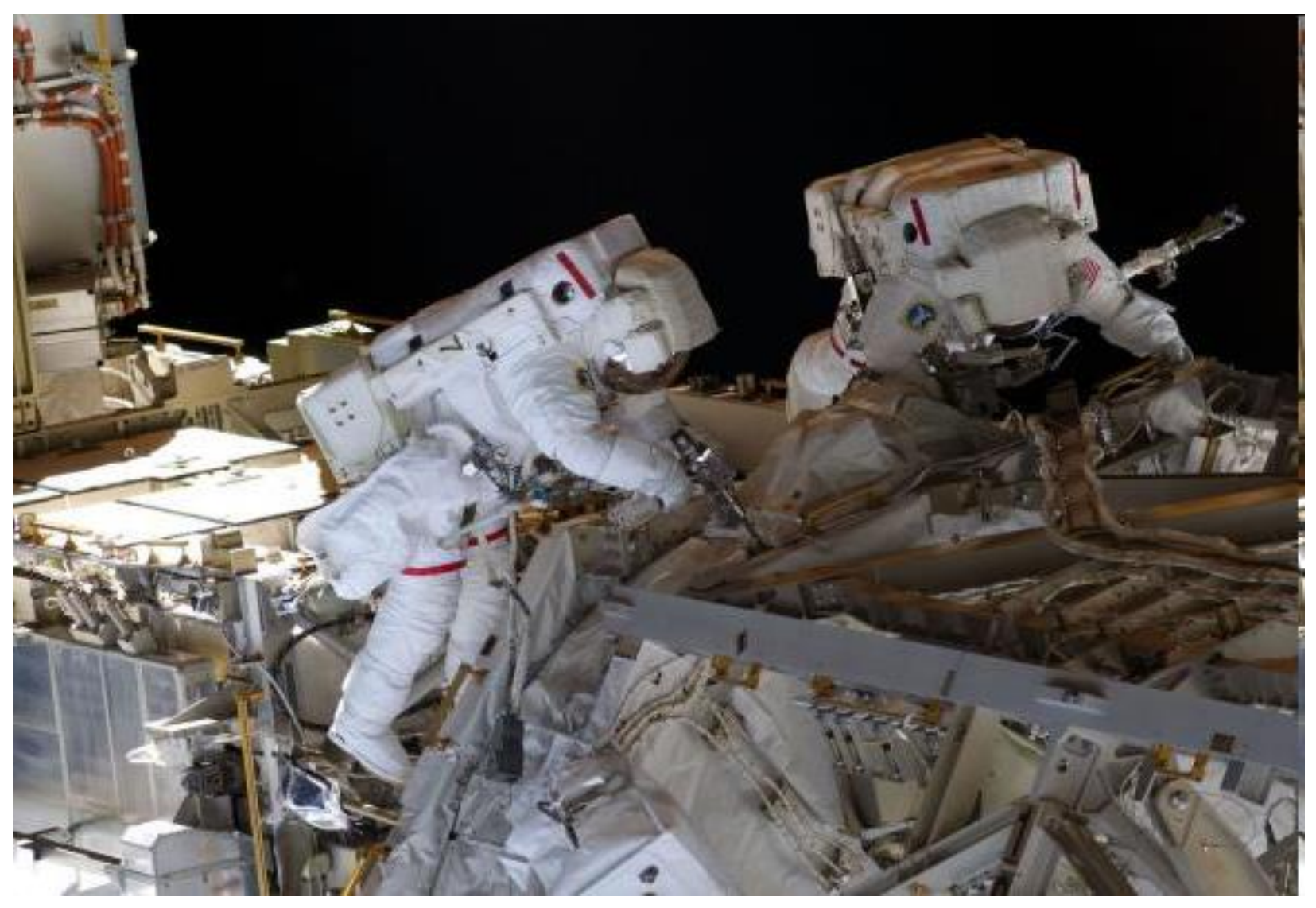

Motor current and vibe levels returned to near normal - rose slightly through the years but still well below safe levels 